\title{
Sequencing Systemic Therapy Pathways for Advanced Hepatocellular Carcinoma: A Cost Effectiveness Analysis
}

\author{
Christopher Sherrow $^{a} \quad$ Kristopher Attwood $^{b}$ Kehua Zhou $^{c}$ \\ Sarbajit Mukherjee $^{d}$ Renuka lyer ${ }^{d}$ Christos Fountzilas $^{d}$ \\ a Department of Pharmacy, Roswell Park Comprehensive Cancer Center, Buffalo, NY, USA; \\ ${ }^{b}$ Department of Biostatistics and Bioinformatics, Roswell Park Comprehensive Cancer \\ Center, Buffalo, NY, USA; ${ }^{C}$ Catholic Health System Internal Medicine Training Program, \\ State University of New York at Buffalo, Buffalo, NY, USA; ${ }^{d}$ GI Division, Department of \\ Medicine, Roswell Park Comprehensive Cancer Center, Buffalo, NY, USA
}

\section{Keywords}

Hepatocellular carcinoma · Cost effectiveness analysis · Quality-adjusted life year

\begin{abstract}
Introduction: Hepatocellular carcinoma ( $\mathrm{HCC}$ ) is the most common form of liver cancer worldwide and carries a poor prognosis. Historically, sorafenib was the only available systemic treatment for advanced HCC. However, in recent years, 6 new treatments have been approved by the US Food and Drug Administration (FDA): regorafenib, lenvatinib, cabozantinib, pembrolizumab, ramucirumab, and nivolumab. Data are lacking regarding the most appropriate sequencing pathway for these agents. Our objective was to conduct a comprehensive cost effectiveness analysis (CEA) of different 1st- and 2nd-line treatment pathways for HCC reflecting all new drug approvals, and then use our data to provide guidance for clinicians on which pathway is the most cost-effective. Materials and Methods: Markov models were used to evaluate the cost effectiveness of 8 different 1 st- and 2 nd-line treatment sequences. The model allowed for 9 possible states. Cost effectiveness ratios (CER) and incremental CER (ICER) were calculated to compare costs between different pathways and against a willingness-topay (WTP) threshold. Efficacy and toxicity data were extracted from the landmark trials for each agent. All agents except ramucirumab were included. The cost of each agent was based on the wholesale acquisition cost (WAC) in USD as of June 2019. Monte-Carlo methods were used to simulate the experience of 1,000,000 patients per treatment sequence for a 12-month period. Results: The pathway with the lowest CER was sorafenib, followed by pembrolizumab (USD 227,741.03/quality-adjusted life year [QALY]). ICER analysis supported implementing
\end{abstract}


2nd-line pembrolizumab-based pathways at a higher WTP threshold of 300,000/quality-adjusted life year. Sensitivity analysis did not substantially change these results. Conclusions: The most cost-effective strategy was 1st-line tyrosine kinase inhibitor therapy followed by 2nd-line immunotherapy. All pathways exceeded a commonly accepted WTP of USD 100150,000/QALY. Our preliminary results warrant further studies to best inform real-world practices.

(C) 2020 The Author(s)

Published by S. Karger AG, Basel

\section{Introduction}

Hepatocellular carcinoma (HCC) is the 4th leading cause of cancer-related death with an estimated 5-year survival of 18\% [1] and a rising incidence in the USA [2]. The era of systemic pharmacotherapy for the treatment of advanced or unresectable HCC began with the US Food and Drug Administration (FDA) approval of the oral multitarget tyrosine kinase inhibitor (TKI) sorafenib, based on the landmark phase III SHARP trial [3]. Following this initial approval of sorafenib, there was a dearth of new therapies for nearly a decade, underscoring the inherent difficulty of treating this deadly cancer. Many other agents failed to demonstrate noninferiority or superiority to sorafenib as 1st-line therapy or did not increase the survival rate as 2nd-line therapies [4-10]. However, starting with the FDA approval of the TKI regorafenib [11] in 2017, 5 other drugs have demonstrated efficacy in HCC and were approved in the following 2 years: the TKIs lenvatinib [12] and cabozantinib [13], the immune checkpoint inhibitors (ICI) pembrolizumab [14] and nivolumab [15] and the anti-vascular endothelial growth factor receptor (VEGFR)-2 monoclonal antibody ramucirumab [16]. With the plethora of systemic choices now available for the treatment of HCC, health care decision-makers, including patients, clinicians, hospitals, private health systems, and public payers (e.g., Medicare) face a new quandary, namely, how can we ideally sequence these agents?

Due to variability across patient populations, it is difficult to directly compare the results of each landmark HCC trial. Given rising health care costs in the USA, there has been a renewed drive to examine the relative cost effectiveness of many cancer therapies and understand the value of each treatment. We performed a cost effectiveness analysis (CEA) for systemic therapy in advanced HCC using different 1st- and 2nd-line scenarios, taking into account the usual toxicities associated with each class of medication. We used Markov modeling, ideal for environments involving sequential, stochastic decisions over time including cancer treatment, and we present a framework for modeling medical decision-making [17].

\section{Methods}

\section{Data Collection}

Sorafenib and lenvatinib are the only two 1st-line FDA-approved systemic therapies; nivolumab, pembrolizumab, cabozantinib, regorafenib, and ramucirumab were all studied and approved for use after 1st-line sorafenib. To conduct our analysis, we performed a comprehensive literature search for all landmark published studies. We extracted the efficacy and toxicity data of these agents from the corresponding published trials of each medication that led to their approval by the FDA. All agents were included, with the exception of ramucirumab (Table 1). Although hypertension is a common adverse drug event (ADE) associated with TKIs, we excluded this particular ADE from our analysis as most patients are asymptomatic (and thus do not have a significantly impaired quality of life), and the condition is usually well-controlled with inexpensive antihypertensive agents such as angiotensin-converting enzyme (ACE) inhibitors and calciumchannel blockers [18]. Ramucirumab was evaluated in the REACH-2 trial [16] and was subsequently approved by the FDA as a 2 nd-line therapy only for patients with $\alpha$-fetoprotein (AFP) $\geq 400 \mathrm{ng} / \mathrm{mL}$. Ramucirumab was excluded from our analysis for 2 reasons. First, unlike all other FDA-approved therapies, its 


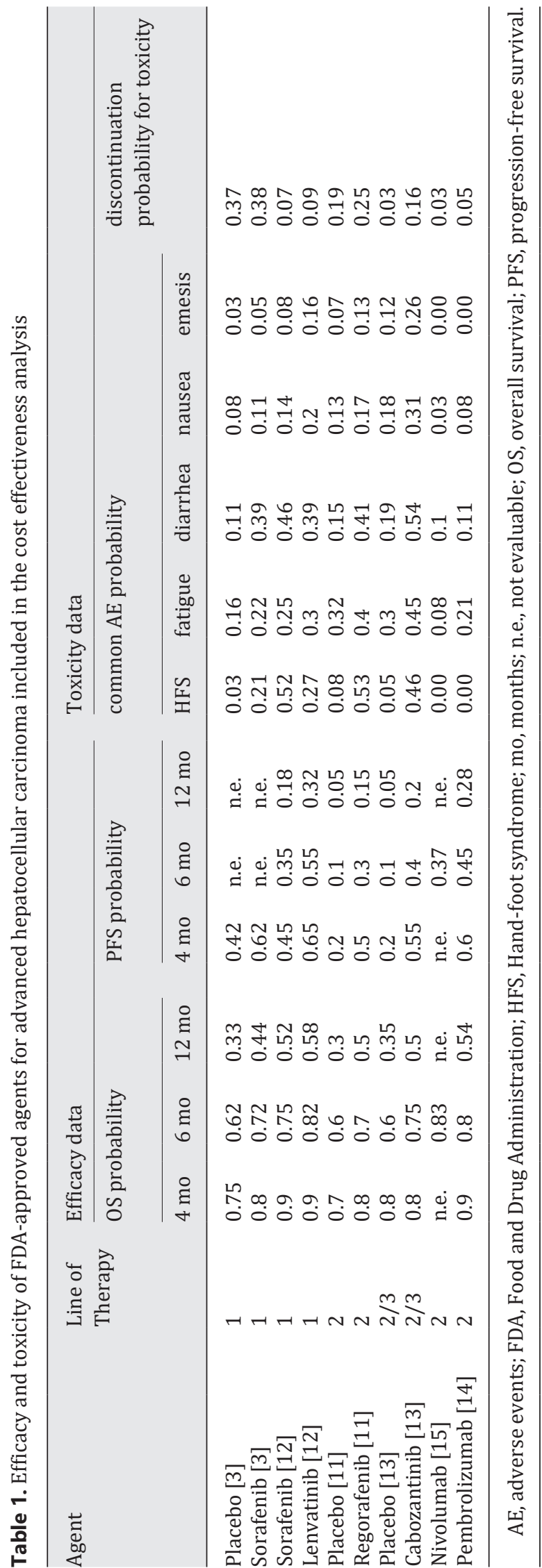




\begin{tabular}{ll|l}
\cline { 2 - 2 } Liver Cancer 2020;9:549-562 \\
\cline { 2 - 2 } & \multicolumn{2}{l}{$\begin{array}{l}\text { DOI: 10.1159/000508485 } 2020 \text { The Author(s). Published by S. Karger AG, Basel } \\
\text { www.karger.com/lic }\end{array}$} \\
\cline { 2 - 3 }
\end{tabular}

Sherrow et al.: Cost Effectiveness of Systemic Therapies for Advanced HCC



Fig. 1. Decision model. The model allows for 9 possible states.

approval is based on the AFP level and, as such, cannot be broadly compared with other agents that do not have this stipulation; second, its dosing is weight-based and thus introduces price variability, limiting extrapolation.

Combinations with breakthrough FDA designation such as bevacizumab and atezolizumab, or lenvatinib and pembrolizumab, were not considered either. When not clearly reported in the text, overall survival (OS) and progression-free survival (PFS) probabilities were extracted by visual inspection of the KaplanMeier curves. To minimize the potential risk of error, 3 of the authors independently performed a visual inspection and their results were averaged. Drug costs were based on wholesale acquisition cost (WAC) [19], listed in USD, and defined as the price a manufacturer charges the wholesaler or distributor to purchase the drug, as of June 2019. Drug costs were estimated based on 1 year of therapy. Lenvatinib is FDA-approved for HCC at 2 initial dose levels: $12 \mathrm{mg}$ once daily for patients $\geq 60 \mathrm{~kg}$ of actual body weight, and $8 \mathrm{mg}$ once daily for patients $<60 \mathrm{~kg}$ of actual body weight. For the purpose of our analysis, the 12-mg dose was selected. Dosing of other agents was established per standard of care as follows: pembrolizumab, a 200-mg flat dose intravenously (i.v.) every 3 weeks; nivolumab, a 240 -mg flat dose i.v. every 2 weeks; sorafenib, $400 \mathrm{mg}$ orally twice daily; regorafenib, $160 \mathrm{mg}$ orally for 21 days of a 28-day treatment cycle; and cabozantinib $60 \mathrm{mg}$ orally once daily.

\section{Model Development}

A Markov modeling approach was utilized to evaluate the cost effectiveness of different 1st- and 2nd-line treatment sequences. The model allowed for 9 possible states (Fig. 1) and the transition probabilities (in 1-month increments) were derived from the extracted study data (Table 1). For treatments with multiple data sources, weighted averages were used to obtain estimates of toxicity and PFS and OS rates.

For the 1st-line treatment, the states included: treatment (1), treatment with toxicity (2), discontinuation due to toxicity (3), progression (4), and death (5). Patients in state 1 could move to any of the 5 states; patients in state 2 could move to any other state; patients in states 3 and 4 transitioned to the 2nd-line treatment (states 6-8) or to state 5; and patients in state 5 (death) remained there. For the 2nd-line treatment, the states included: treatment (6), treatment with toxicity (7), discontinuation due to toxicity (8), progression 


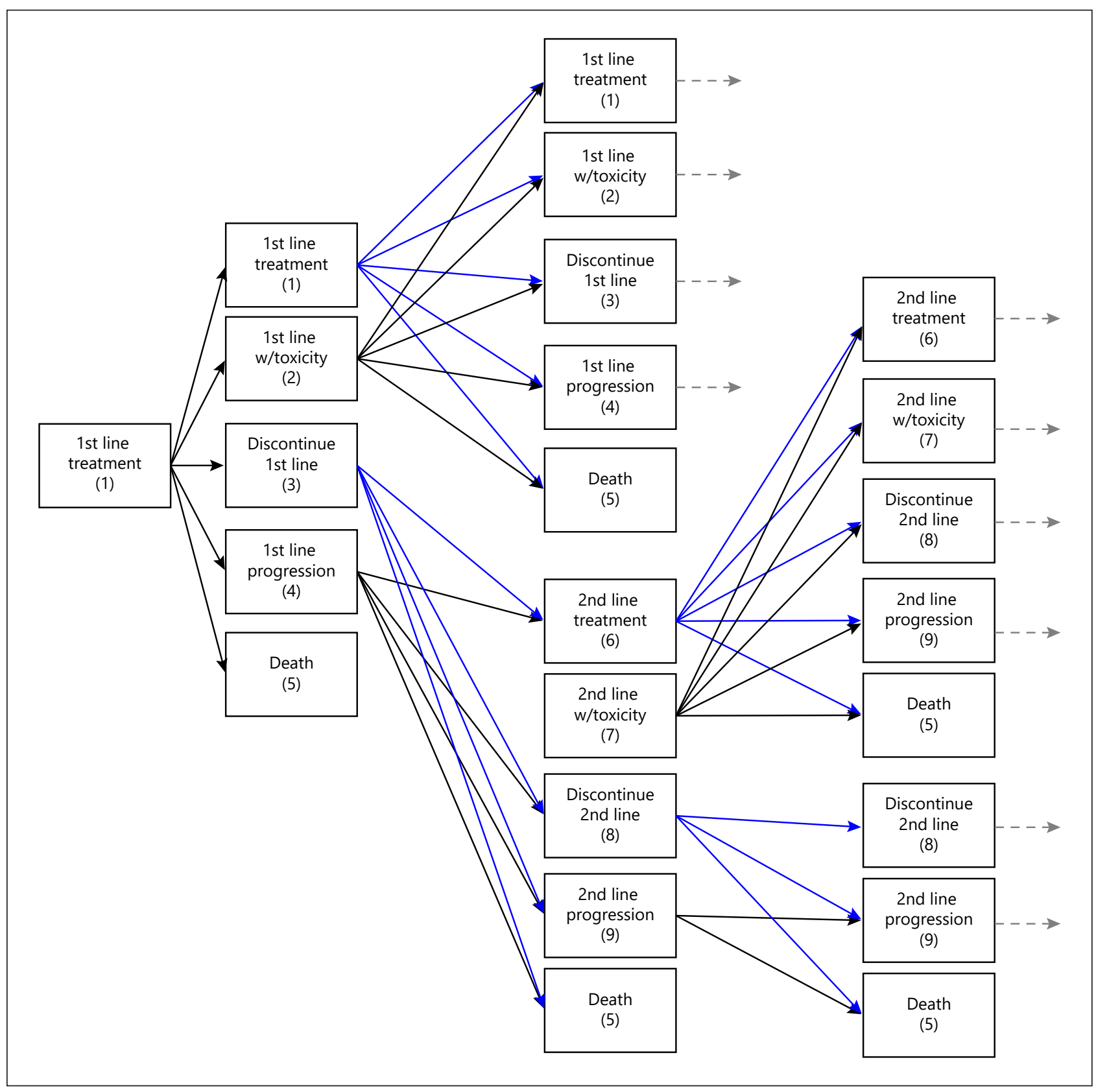

Fig. 2. Model description.

(9), and death (5). Patients in state 6 could move to any of the 5 states (5-9); patients in state 7 could move to any other state; patients in state 8 could remain in that state or transition to progression (9) or death (5); patients in state 9 could remain there or transition to death (5); and patients in state 5 (death) remained there (Fig. 2).

The cost of being in a treatment state is dependent on the current therapy. A health utility was assigned to each treatment state: patients on treatment received a mean health utility (MHU) of 0.84 , patients who progressed received a MHU of 0.50 , patients who had toxicity received a MHU of 0.70 , and patients who died received a MHU of 0 . The MHU values are consistent with the results of Le [20], and the observed health utility for a given patient was randomly generated using $\beta$ distribution based on the MHU for a given state. Qualityadjusted life years (QALYs) were then obtained by summing across all treatment states the product of the state's healthy utility and the time in the given state. 


\begin{tabular}{l|l}
\hline Liver Cancer 2020;9:549-562 \\
\hline DOI: 10.1159/000508485 & $\begin{array}{l}\text { @ 2020 The Author(s). Published by S. Karger AG, Basel } \\
\text { www.karger.com/lic }\end{array}$ \\
\hline
\end{tabular}

Table 2. Important definitions

\begin{tabular}{ll}
\hline QALY & $\begin{array}{l}\text { Quality-adjusted life year, referring to the number of quality life years accumulated by a patient. } \\
\text { If a patient was on treatment (with no toxicity or progression) for the entire simulated year, then } \\
\text { QALY = 1; if a patient died during the first month, then QALY = 0; if a patient experienced } \\
\text { toxicity or progression, then QALY will range between } 0 \text { and } 1\end{array}$ \\
\hline Cost & The accumulated cost based on the treatment received \\
\hline CER & $\begin{array}{l}\text { Cost effectiveness ratio, i.e., the ratio of the average cost relative to the average QALY; essentially, this } \\
\text { quantity represents the cost (in USD) required to achieve } 1 \text { quality-of-life year }\end{array}$ \\
\hline ICER & $\begin{array}{l}\text { Incremental cost effectiveness ratio, which represents the incremental cost of providing } 1 \text { additional } \\
\text { QALY for one treatment versus another }\end{array}$ \\
\hline Toxicity rate & The percentage of simulated patients that experienced a toxicity at some point during treatment \\
\hline Average survival & The average survival time (in months) after the simulated year \\
\hline
\end{tabular}

Table 3. Cost effectiveness of different treatment pathways for advanced hepatocellular carcinoma

\begin{tabular}{|c|c|c|c|c|c|c|c|}
\hline Regimen & $\begin{array}{l}\text { Treatments: } \\
\text { 1. = 1st-line } \\
2 .=2 \text { nd-line }\end{array}$ & $\begin{array}{l}\text { Average } \\
\text { QALY }\end{array}$ & $\begin{array}{l}\text { Average } \\
\text { cost, USD }\end{array}$ & CER & $\begin{array}{l}\text { Toxicity } \\
\text { rate }\end{array}$ & $\begin{array}{l}\text { Average } \\
\text { survival, } \\
\text { months }\end{array}$ & $\begin{array}{l}12 \text {-Month } \\
\text { survival } \\
\text { rate }\end{array}$ \\
\hline A & $\begin{array}{l}\text { 1. sorafenib } \\
\text { 2. regorafenib }\end{array}$ & 0.549 & $145,018.96$ & $264,185.19$ & 0.459 & 8.62 & 0.515 \\
\hline B & $\begin{array}{l}\text { 1. sorafenib } \\
\text { 2. cabozantinib }\end{array}$ & 0.556 & $148,669.18$ & $267,527.62$ & 0.461 & 8.70 & 0.534 \\
\hline $\mathrm{C}$ & $\begin{array}{l}\text { 1. sorafenib } \\
\text { 2. pembrolizumab }\end{array}$ & 0.566 & $128,903.33$ & $227,741.03$ & 0.386 & 8.71 & 0.535 \\
\hline $\mathrm{D}$ & $\begin{array}{l}\text { 1. sorafenib } \\
\text { 2. nivolumab }\end{array}$ & 0.573 & $136,397.20$ & $238,070.23$ & 0.372 & 9.09 & 0.597 \\
\hline $\mathrm{E}$ & $\begin{array}{l}\text { 1. lenvatinib } \\
\text { 2. regorafenib }\end{array}$ & 0.597 & $152,919.47$ & $256,008.73$ & 0.424 & 9.12 & 0.574 \\
\hline $\mathrm{F}$ & $\begin{array}{l}\text { 1. lenvatinib } \\
\text { 2. cabozantinib }\end{array}$ & 0.602 & $155,529.84$ & $258,460.32$ & 0.426 & 9.18 & 0.587 \\
\hline G & $\begin{array}{l}\text { 1. lenvatinib } \\
\text { 2. pembrolizumab }\end{array}$ & 0.610 & $140,419.08$ & $230,371.17$ & 0.369 & 9.19 & 0.590 \\
\hline $\mathrm{H}$ & $\begin{array}{l}\text { 1. lenvatinib } \\
\text { 2. nivolumab }\end{array}$ & 0.615 & $146,352.27$ & $237,899.15$ & 0.358 & 9.48 & 0.638 \\
\hline
\end{tabular}

QALY, quality-adjusted life year; CER, cost effectiveness ratio.

Monte-Carlo methods were used to simulate the experience of 1,000,000 patients per treatment sequence for a 12-month period. The patients moved through different states based on the derived transition matrices, and accumulated costs and QALYs were based on the states they experienced.

\section{Outcome Measures}

The following quantities describe the operating characteristics of the different treatment sequences and were calculated from the simulated data (see Table 2 for definitions): QALY, cost, cost effectiveness ratio (CER, representing the cost required to achieve 1 quality-of-life year), toxicity rate and average survival. Additionally, the cost effectiveness was compared between treatment groups using the incremental CER (ICER). 
Sherrow et al.: Cost Effectiveness of Systemic Therapies for Advanced HCC

Table 4. Comparing incremental cost effectiveness ratios of different treatment pathways for advanced hepatocellular carcinoma and assessing the net benefit

\begin{tabular}{|c|c|c|c|c|c|c|c|}
\hline \multirow[t]{2}{*}{ Regimen } & \multirow[t]{2}{*}{ Cost, USD } & \multirow[t]{2}{*}{ QALYs } & \multicolumn{3}{|c|}{ ICERs compared to } & \multicolumn{2}{|l|}{ Net benefit } \\
\hline & & & $\begin{array}{l}\text { lowest } \\
\text { cost (C) }\end{array}$ & $\begin{array}{l}\text { next lowest } \\
\text { cost }\end{array}$ & regimen $\mathrm{D}^{\mathrm{c}}$ & $\begin{array}{l}\text { USD 150,000/ } \\
\text { QALY }\end{array}$ & $\begin{array}{l}\text { USD 300,000/ } \\
\text { QALY }\end{array}$ \\
\hline $\mathrm{C}$ & $128,903.33$ & 0.566 & - & - & - & $-44,003.33$ & $40,896.67$ \\
\hline $\mathrm{D}$ & $136,397.20$ & 0.573 & $1,070,553.0$ & $1,070,553.0$ & - & $-50,447.20$ & $35,502.80$ \\
\hline $\mathrm{G}$ & $140,419.08$ & 0.610 & $261,721.6$ & $108,699.5$ & $108,699.5$ & $-48,919.08$ & $42,580.92$ \\
\hline $\mathrm{H}$ & $146,352.27$ & 0.615 & $356,100.8$ & 1,186,638.0 & $237,025.48$ & $-54,102.27$ & $38,147.73$ \\
\hline $\mathrm{E}$ & $152,919.47$ & 0.597 & $774,714.2$ & $\mathrm{SD}^{\mathrm{b}}$ & - & $-63,369.47$ & $26,180.53$ \\
\hline $\mathrm{F}$ & $155,529.84$ & 0.602 & $739,625.3$ & $\mathrm{SD}^{\mathrm{b}}$ & - & $-65,229.84$ & $25,070.16$ \\
\hline A & $145,018.96$ & 0.549 & dominated $^{\mathrm{a}}$ & - & - & - & - \\
\hline B & $148,669.18$ & 0.556 & dominated $^{\mathrm{a}}$ & - & - & - & - \\
\hline
\end{tabular}

Sensitivity Analysis

A sensitivity analysis was conducted to evaluate the impact of the subjective QALY measure on the operating characteristics of the treatment sequences. The QALY weights were reduced and variability in the $\beta$ distributions was increased (i.e., less consistency in QALY experienced between patients).

\section{Results}

Two 1st-line therapies and four 2nd-line therapies were included in the analysis, which resulted in the evaluation of 8 different treatment sequences. Table 3 provides the outcome measures of interest for each treatment pathway.

The CERs for 1st-line TKI therapy followed by 2nd-line TKI therapy all exceeded the CERs for 1st-line TKIs followed by 2nd-line ICI. The sequence with the overall lowest CER was sorafenib followed by pembrolizumab (USD 227,741.03/QALY). The next most cost-effective option was lenvatinib followed by pembrolizumab, which had a similar CER of 230,371.17 USD/QALY. Comparing 1st-line lenvatinib and sorafenib, lenvatinib appears to be slightly more cost-effective as all the CERs for pathways that included lenvatinib were lower than the comparable sorafenib pathways, except for sorafenib and pembrolizumab.

Comparing 2nd-line nivolumab and pembrolizumab, pembrolizumab appeared slightly more cost-effective as all its CERs were lower than the corresponding nivolumab pathways. For example, lenvatinib followed by pembrolizumab yielded a CER of USD 230,371.17 versus USD $237,899.15$ for lenvatinib followed by nivolumab. However, it is important to note that the difference in CER between different immunotherapy pathways is not large. While lenvatinib followed by nivolumab yielded a CER of USD 237,899.15/QALY, sorafenib followed by nivolumab had a CER of USD 238,070.23/QALY. Both of these CERs exceeded those for the corresponding pembrolizumab pathways, but the difference between these 2 is only USD 171.08/QALY. Similarly, the difference in CER between sorafenib followed by pembrolizumab and lenvatinib followed by pembrolizumab is USD 2,630.14/QALY in favor of the sorafenibbased pathway.

Decreased cost effectiveness to 1st-line TKI and 2nd-line ICI therapy is observed when considering only 1st- and 2nd-line TKI therapies. While comparable to each other, notably, 
Table 5. Sensitivity analysis considering different QALY weights

\begin{tabular}{l|l}
\hline DOI: $10.1159 / 000508485$ & $\begin{array}{l}\text { C } 2020 \text { The Author(s). Published by S. Karger AG, Basel } \\
\text { www.karger.com/lic }\end{array}$ \\
\hline
\end{tabular}

Sherrow et al.: Cost Effectiveness of Systemic Therapies for Advanced HCC

\begin{tabular}{lcc}
\hline $\begin{array}{l}\text { Treatments: } \\
\text { 1. }=\text { st-line }\end{array}$ & $\begin{array}{l}\text { Average } \\
\text { QALYs }\end{array}$ & \multicolumn{1}{l}{ CER } \\
2. =2nd-line & 0.511 & $283,794.44$ \\
\hline $\begin{array}{l}\text { 1. Sorafenib } \\
\text { 2. Regorafenib }\end{array}$ & 0.517 & $287,561.28$ \\
\hline $\begin{array}{l}\text { 1. Sorafenib } \\
\text { 2. Cabozantinib }\end{array}$ & 0.527 & $244,598.35$ \\
$\begin{array}{l}\text { 1. Sorafenib } \\
\text { 2. Pembrolizumab }\end{array}$ & 0.532 & $256,385.71$ \\
\hline $\begin{array}{l}\text { 1. Sorafenib } \\
\text { 2. Nivolumab }\end{array}$ & 0.557 & $274,541.24$ \\
\hline $\begin{array}{l}\text { 1. Lenvatinib } \\
\text { 2. Regorafenib }\end{array}$ & 0.561 & $277,236.79$ \\
\hline $\begin{array}{l}\text { 1. Lenvatinib } \\
\text { 2. Cabozantinib }\end{array}$ & & \\
\hline $\begin{array}{l}\text { 1. Lenvatinib } \\
\text { 2. Pembrolizumab }\end{array}$ & 0.570 & $246,349.26$ \\
\hline $\begin{array}{l}\text { 1. Lenvatinib } \\
\text { 2. Nivolumab }\end{array}$ & 0.573 & \\
\hline
\end{tabular}

CER, cost effectiveness ratio; QALY, quality-adjusted life years.

the CERs are all higher than those for 1st-line TKI followed by 2nd-line ICI therapy. Comparing 2nd-line regorafenib and cabozantinib, regorafenib appears slightly more cost-effective. The CER of sorafenib followed by regorafenib (USD 264,185.19/QALY) was lower that of sorafenib followed by cabozantinib (USD 267,527.62/QALY). Likewise, the CER of lenvatinib followed by regorafenib was also lower than that of lenvatinib followed by cabozantinib (USD 256,008.73/QALY vs. 258,460.32/QALY, respectively).

If considering only oral therapies, the pathway of lenvatinib followed by regorafenib appears to be the most cost-effective as it has the lowest CER (USD 256,008.73/QALY). However, this is still significantly higher than any 1st-line TKI and 2nd-line ICI pathway. The least cost-effective pathway in our analysis was sorafenib followed by cabozantinib, which yielded the highest CER of USD 267,527.62/QALY.

ICERs, defined as the difference in average cost between pathways divided by the difference in average QALY between pathways, were also calculated (Table 4). In accordance with traditional CEAs, these ICER values between pathways were compared against a predefined willingness-to-pay (WTP) threshold to determine relative cost effectiveness. Importantly, all the pathways compared are relevant treatment options, but differ in cost effectiveness. Table 4 summarizes appropriate pairwise comparisons; ICERs for each regimen were compared to the lowest-cost pathway and the next lowest-cost pathway. Pathways A (sorafenib followed by regorafenib) and B (sorafenib followed by cabozantinib) both had higher costs and fewer QALY compared to the lower-cost and more efficacious pathway C (sorafenib followed by pembrolizumab), and hence are dominated by C. Pathway D had an ICER of USD 1,070,553.0/QALY compared to Pathway C. However, since the ICER of moving from Pathway D to the more costly Pathway G falls, the lower-cost pathway of D is dominated. Thus, it is most appropriate to compare Pathways G and H to Pathway C. Pathway G offered an ICER of USD 261,721.6/QALY compared to Pathway C, which far exceeds the commonly 
accepted WTP threshold of USD 100,000/QALY. Pathway H had an ICER of USD 356,100.8/ QALY compared to Pathway $C$, which again exceeds the above WTP threshold. Using this threshold as a rigid decision point, a decision-maker would not implement either pathway as they are not considered cost-effective. When faced with multiple alternatives, it is also useful to calculate the net benefit of each pathway. This was calculated by using 2 WTP thresholds. Assuming a WTP of USD 150,000/QALY, none of the pathways would be implemented as the net benefit for each pathway did not exceed 0 . However, if a higher WTP threshold of USD 300,000 USD/QALY was used, the pathway G (lenvatinib followed by pembrolizumab) is most likely to be implemented as it offered the highest net benefit (USD 42,580.92). In other words, at a WTP threshold of USD 300,000/QALY, the pathway of lenvatinib followed by pembrolizumab was the most cost-effective. Pathway C (sorafenib followed by pembrolizumab) is the next most likely to be implemented as its net benefit was second highest at USD $40,896.67$. The net benefit for 2 nd-line immunotherapy-based pathways all exceeded that for 2nd-line TKI-based therapies at a WTP threshold of USD 300,000/QALY. Overall, these results illustrate the dramatically high costs of HCC treatment, and suggest that a higher WTP threshold is needed.

In the sensitivity analysis, when considering less impactful and more variable QALY weights (Table 5), the relative improvement in CER for pembrolizumab versus other 2nd-line treatments was slightly reduced. However, the pathway of sorafenib followed by pembrolizumab still yielded the lowest CER (USD 244,598.35/QALY), followed by lenvatinib and pembrolizumab (USD 246,349.26/QALY). Thus, the general conclusions of the primary analysis were upheld by the sensitivity analysis.

\section{Discussion}

Over the past few years, multiple new agents were approved for the systemic therapy of advanced HCC. There is no direct comparison between the approved 2nd-line agents, with all being compared to placebo, so there are no data to guide the sequencing of available active agents. CEA examines the amount of money required to extend life by 1 year using a certain treatment accounting for quality [21]. To our knowledge, our study is unique in that it attempts to characterize the cost effectiveness of modern systemic HCC therapy in the continuum of care. Our results indicate that the most cost-effective 1st- and 2nd-line pathway is a TKI followed by ICI. When comparing the 2 ICI options, pembrolizumab appeared to be slightly more cost-effective than nivolumab. This is demonstrated by the pembrolizumab-based pathways (Pathways G and C) having the highest and second-highest net benefit for a WTP threshold of USD 300,000/QALY. When considering 2nd-line TKI options (e.g., cabozantinib or regorafenib), regorafenib appears slightly more cost-effective with lenvatinib or sorafenib as the 1st-line therapy.

Notably, as demonstrated by the ICER values (Table 4), none of the studied pathways would be deemed cost-effective based on the WTP threshold in the USA in the range of USD 100,000-150,000/QALY for oncologic therapies [22]. The relevant ICER values calculated all exceeded this threshold. Additionally, Azimi and Welch [23] found that researchers generally opposed implementing interventions if the ICER was greater than USD 166,000/QALY gained, and there was ambiguity about cost effectiveness if the ICER was in the range of USD 61,500166,000 USD/QALY gained. Some researchers favored implementing the intervention, some did not favor it, or else a clear conclusion could not be identified for the above range; the authors thus determined it to be a "gray area," where researchers arrived at different conclusions, even when the ICERs are similar. Nevertheless, many antineoplastic agents are commonly used despite exceeding this threshold. For example, bevacizumab in colon cancer 
has an ICER greater than USD $>350,000 /$ QALY in the 1st- and 2nd-line settings [24]. Our study suggests that if a higher WTP threshold of USD 300,000/QALY is used, the pathway of lenvatinib followed by pembrolizumab is the most cost-effective as it offers the most net benefit. In general, a greater net benefit was achieved with 2nd-line immunotherapy-based regimens. Our study also clearly outlines the high costs of treatment for advanced HCC and illustrates the dire need for more cost-effective therapies.

Although other CEAs have been performed for pharmacotherapy treatment of HCC (lenvatinib, [25], sorafenib [26], regorafenib [27, 28], and cabozantinib [29]), our study is unique with its focus on sequencing available agents, rather than evaluating active treatment versus no treatment or comparing the 2 available 1st-line options [25]. In a study of a CEA of lenvatinib versus sorafenib for advanced HCC, a partitioned-survival model was developed to estimate the cost effectiveness when treating patients with HCC over a lifetime horizon and considering total public healthcare expenditure [25]. The incremental cost was USD 3,827 and the incremental life years and QALYs were 0.27 and 0.23 , respectively, for lenvatinib versus sorafenib. Similar to our results, the authors concluded that lenvatinib was costeffective when compared with sorafenib as the 1st treatment of unresectable HCC in Japan. Compared to best supportive care, the CER for sorafenib ranges between USD 101,399.11 and 224,914/QALY [26]. The CER of regorafenib is higher (range USD 201,797-268,506/QALY) $[27,28]$, while for cabozantinib it is USD 1,040,675/QALY based on the results of the CELESTIAL study [29]. For all agents, the cost is higher than the WTP threshold. An exception is dose-adjusted sorafenib with CER 45,584.88 USD/QALY [26]. Though a reduced versus a standard dose of sorafenib has not been formally evaluated in prospective studies, in a retrospective study using data from Veteran Affairs (VA) Administration hospitals, the cost was also lower, and, using propensity score matching analysis, there was no significant difference in OS (adjusted hazards ratio [HR] 0.92; 95\% confidence interval [CI] 0.83-1.01). Similarly, body weight-based dosing regimens can be more cost-effective while maintaining the clinical efficacy of ICI [30, 31].

Our study has several limitations. Foremost, data on efficacy and toxicity parameters were extracted from corresponding published trials; besides heterogeneity between trial designs and patient populations, head-to-head comparisons between drug treatments is still lacking. Importantly, cabozantinib was tested in both the 2nd- and 3rd-line setting in the CELESTIAL study (27\% of patients had received 2 prior lines of therapy) [13].

Second, single-center cost estimates and WAC pricing models may limit the generalizability of our results. Traditionally, most CEAs use WAC pricing to approximate drug costs [32]. WAC pricing is an estimate of the manufacturer's list price for a drug to wholesalers or direct purchasers with no discounts or rebates. The actual cost of the drug usually differs from the WAC pricing. In the real world, rebates and discounts are often offered to pharmacy benefits managers (PBMs) on behalf of payers in proprietary negotiations. Meanwhile, price negotiations between PBMs and pharmaceutical companies are influenced by market forces, such as market share, patient population, the availability of generic drugs, and in-class competition specific to certain drugs. This lack of transparency can make the estimation of true drug costs problematic. As an example, Mattingly et al. [33] compared WAC pricing data to the discounted prices observed by the US VA Department. Oncology drugs (including bortezomib, pembrolizumab, ramucirumab, and pemetrexed) demonstrated the smallest discounts from WAC pricing to the discounted VA price (ranging from 26 to 30\%) amongst all drug classes considered.

Third, several costs were not accounted for in our analysis, including the substantial costs associated with treating underlying cirrhosis, adverse drug events (e.g., admission for immune-mediated reactions, intractable nausea/vomiting, etc.) and supportive care measures. In order to simplify the analysis and broaden the potential application of results, an- 
cillary costs associated with intravenous therapies (e.g., institutional infusion costs) were not considered either. Costs per infusion hour can vary. One microcosting study estimated a cost of USD 654 (administrative and dispensing costs) for a 203-min infusion of antineoplastic therapy and USD 409 for a 103-min infusion of the same therapy at a US-based infusion center [34]. These additional costs can negatively impact the cost effectiveness of the immunotherapy options compared to oral therapies. Of note, pembrolizumab and nivolumab should have the same infusion cost, as they are both given as a 30-min infusion. Ramucirumab is also an intravenous infusion, but it was not included in our analysis due to reasons stated in the Methods section.

Finally, our sequencing pathways consisted only of 1st- and 2nd-line estimates of costs. In real life, patients with Child Pugh Class B cirrhosis are treated as long as their liver functions and ECOG performance status are in line with the guidance in the package insert, so the duration of their therapy may not be as long as that for Child Pugh A patients (who were the trial candidates). However, it is possible that patients retaining a good performance status and preserved organ function may qualify for 3rd-line therapy (e.g., sorafenib followed by pembrolizumab followed by cabozantinib). Our analysis did not consider the increased costs associated with 3rd-line therapy and beyond. It is also important to acknowledge that cabozantinib, regorafenib, pembrolizumab, and nivolumab are not FDA-approved for use after 1st-line lenvatinib as they were studied only in the post-sorafenib setting; the HCC clinician must exercise clinical judgment in deciding whether to prescribe these agents in the postlenvatinib setting.

After the data completion of our analysis, new data regarding the immunotherapy options was announced. The confirmatory phase III KEYNOTE-240 trial for pembrolizumab did not meet coprimary end points as pembrolizumab failed to improve PFS and OS in advanced HCC patients previously treated with systemic therapy [35]. Although it appears that pembrolizumab did improve OS versus placebo, this was not statistically significant per the prespecified statistical plan (HR 0.78; 95\% CI 0.611-0.998; $p=0.0238$ ). Pembrolizumab also trended towards improved PFS, but this again did not reach statistical significance (HR 0.78; $p=$ $0.0219)$. The implications of this trial remain unclear, and as of writing this paper, pembrolizumab remains FDA-approved for the treatment of HCC. Additionally, on 24 June 2019, Bristol-Myers Squibb announced the results of CheckMate-459, a randomized phase III study evaluating nivolumab versus sorafenib as 1 st-line treatment in patients with unresectable HCC. This study was then subsequently presented at the 2019 European Society for Medical Oncology (ESMO) meeting on 27 September 2019. It was noted that OS did not meet the predefined threshold of statistical significance (HR 0.84, $p=0.0419$ ). The median OS was 16.4 months for nivolumab and 14.7 months for sorafenib (HR 0.85; 95\% CI 0.72-1.02; $p=0.0752$ ) [36]. The full trial texts of CheckMate-459 and KEYNOTE-240 were unavailable at the time of manuscript submission. Our CEA did not consider the use of nivolumab as a 1st-line therapy, and thus these new data do not change our conclusions. The impact on OS from subsequent therapies is not yet known and will be helpful in future CEAs.

Beyond the costs, there are patient-specific factors that may guide the clinician in therapy selection. The advent of next-generation sequencing (NGS) technologies has allowed for a detailed molecular profiling of tumor types and may play a critical role in selecting an appropriate treatment pathway. For example, WNT/ $\beta$-catenin pathway and TP53 alterations are common in HCC and are indicative of mutually exclusive molecular subsets [37]. Mutations activating the PI3K/mTOR pathway were associated with poor outcomes in patients treated with sorafenib [37]. In contrast, mutations predicted to activate the WNT pathway were associated with innate resistance to immune checkpoint blockade alterations, precluding the use of nivolumab or pembrolizumab [37]. Genomic profiling costs were not in our analysis, but the use of biomarkers could potentially reduce overall costs by avoiding unnecessary ther- 
apies. Additionally, lenvatinib appears to be superior for patients with hepatitis B-related HCC, while sorafenib with its immunomodulatory effects appears to be superior for patients with hepatitis C-related HCC where immune mechanisms drive the carcinogenesis. Furthermore, radiographic responses are more frequent with immunotherapy and lenvatinib, and in patients who are highly symptomatic (i.e., with pain or portal vein thrombus) these may be preferable.

The adverse event profile of the agents also warrants close consideration. For example, a clinician may favor immunotherapy for an older patient with a diminished performance status, given the higher rates of fatigue observed with TKI therapy. Patients with coronary artery disease or recent variceal bleed may not be ideal candidates for antiangiogenic therapies. Lastly, the convenience of patients taking oral therapies at home versus the necessity of infusion center visits for intravenous therapies may also affect treatment selection.

In summary, our CEA generally favored the approach of using a TKI as the 1st-line therapy followed by immunotherapy as the 2nd-line therapy as the most cost-effective pathway for the treatment of advanced HCC. Pembrolizumab appeared slightly more cost-effective than nivolumab, but not greatly so. Using either regorafenib or cabozantinib as the 2nd-line therapy appeared to be a less cost-effective strategy than ICI. These results were upheld by the sensitivity analysis.

As mentioned before, our analysis also illustrates that the costs of HCC treatment are exceptionally high and would not be considered cost-effective with a WTP threshold of USD 100,000-150,000/QALY. Our intention was to outline the available treatment options and provide a preliminary discussion regarding the difficult issue of cost effectiveness among the plethora of HCC therapies now available. While we acknowledge several important limitations of our study, we hope it provides guidance to clinicians and the impetus for future studies in this area. Further analysis and studies across multiple centers should be conducted. Real-world phase IV studies and analyses from claims databases will augment our assessment of the "true costs" of care with a concomitant assessment of clinical benefit.

\section{Acknowledgements}

The authors wish to acknowledge Kristyne Hawkes, MS, MBA, Pharmacy Business Manager at Roswell Park Comprehensive Cancer Center for her assistance in gathering and validating price data for the analysis.

\section{Statement of Ethics}

This was a retrospective review of published data and thus ethics approval of subjects was not required.

\section{Disclosure Statement}

No competing financial interests exist. R.I.: consultant Eisai, Merck, Bayer and Exelixis; grant support Merck. C.F.: consultant for AstraZeneca outside of the scope of the submitted work (fees paid to the institute); research support from the National Cancer Comprehensive Cancer Network/Taiho Oncology, Merck, and Pfizer Inc. (paid to institute).

\section{Funding Sources}

Institutional support was via the National Cancer Institute (NCI) grant P30CA016056 for C.S., K.A., S.M., R.I., and C.F. 


\begin{tabular}{l|l|}
\hline Liver Cancer 2020;9:549-562 \\
\hline DOI: 10.1159/000508485 & $\begin{array}{l}\text { @ 2020 The Author(s). Published by S. Karger AG, Basel } \\
\text { www.karger.com/lic }\end{array}$ \\
\hline
\end{tabular}

Sherrow et al.: Cost Effectiveness of Systemic Therapies for Advanced HCC

\section{Author Contributions}

C.S. and C.F. had full access to all the data in the study and take responsibility for the integrity of the data and the accuracy of the data analysis. Study concept and design: C.F. and K.A. Acquisition, analysis, and interpretation of data and drafting of the manuscript: C.S., C.F., and K.A. Statistical analysis: K.A. Study supervision: C.S. and C.F. Critical revision of the manuscript for important intellectual content, and administrative, technical, or material support: all authors.

\section{References}

1 Villanueva A. Hepatocellular Carcinoma. N Engl J Med. 2019 Apr;380(15):1450-62.

2 Howlader N, Noone AM, Krapcho M, Miller D, Brest A, Yu M, et al., editors. [Internet] SEER Cancer Statistics Review (CSR) 1975-2017 Cancer Stat Facts: Liver and Intrahepatic Bile Duct Cancer [cited April 2020]. Bethesda (MD): National Cancer Institute. Available from: https://seer.cancer.gov/csr/1975_2017/

3 Llovet JM, Ricci S, Mazzaferro V, Hilgard P, Gane E, Blanc JF, et al.; SHARP Investigators Study Group. Sorafenib in advanced hepatocellular carcinoma. N Engl J Med. 2008 Jul;359(4):378-90.

4 Cainap C, Qin S, Huang WT, Chung IJ, Pan H, Cheng Y, et al. Linifanib versus Sorafenib in patients with advanced hepatocellular carcinoma: results of a randomized phase III trial. J Clin Oncol. 2015 Jan;33(2):172-9.

5 Cheng AL, Kang YK, Lin DY, Park JW, Kudo M, Qin S, et al. Sunitinib versus sorafenib in advanced hepatocellular cancer: results of a randomized phase III trial. J Clin Oncol. 2013 Nov;31(32):4067-75.

6 Abou-Alfa GK, Niedzwieski D, Knox JJ, Kaubisch A, Posey J, et al. Phase III randomized study of sorafenib plus doxorubicin versus sorafenib in patients with advanced hepatocellular carcinoma (HCC): CALGB 80802 (Alliance). J Clin Oncol. 2016;2019:192.

7 Johnson PJ, Qin S, Park JW, Poon RT, Raoul JL, Philip PA, et al. Brivanib versus sorafenib as first-line therapy in patients with unresectable, advanced hepatocellular carcinoma: results from the randomized phase III BRISK-FL study. J Clin Oncol. 2013 Oct;31(28):3517-24.

8 Zhu AX, Kudo M, Assenat E, Cattan S, Kang YK, Lim HY, et al. Effect of everolimus on survival in advanced hepatocellular carcinoma after failure of sorafenib: the EVOLVE-1 randomized clinical trial. JAMA. 2014 Jul;312(1): 57-67.

9 Llovet JM, Decaens T, Raoul JL, Boucher E, Kudo M, Chang C, et al. Brivanib in patients with advanced hepatocellular carcinoma who were intolerant to sorafenib or for whom sorafenib failed: results from the randomized phase III BRISK-PS study. J Clin Oncol. 2013 Oct;31(28):3509-16.

10 Zhu AX, Rosmorduc O, Evans TR, Ross PJ, Santoro A, Carrilho FJ, et al. SEARCH: a phase III, randomized, doubleblind, placebo-controlled trial of sorafenib plus erlotinib in patients with advanced hepatocellular carcinoma. J Clin Oncol. 2015 Feb;33(6):559-66.

11 Bruix J, Qin S, Merle P, Granito A, Huang YH, Bodoky G, et al.; RESORCE Investigators. Regorafenib for patients with hepatocellular carcinoma who progressed on sorafenib treatment (RESORCE): a randomised, doubleblind, placebo-controlled, phase 3 trial. Lancet. 2017 Jan;389(10064):56-66.

12 Kudo M, Finn RS, Qin S, Han KH, Ikeda K, Piscaglia F, et al. Lenvatinib versus sorafenib in first-line treatment of patients with unresectable hepatocellular carcinoma: a randomised phase 3 non-inferiority trial. Lancet. 2018 Mar;391(10126):1163-73.

13 Abou-Alfa GK, Meyer T, Cheng AL, El-Khoueiry AB, Rimassa L, Ryoo BY, et al. Cabozantinib in Patients with Advanced and Progressing Hepatocellular Carcinoma. N Engl J Med. 2018 Jul;379(1):54-63.

14 Zhu AX, Finn RS, Edeline J, Cattan S, Ogasawara S, Palmer D, et al.; KEYNOTE-224 investigators. Pembrolizumab in patients with advanced hepatocellular carcinoma previously treated with sorafenib (KEYNOTE224): a non-randomised, open-label phase 2 trial. Lancet Oncol. 2018 Jul;19(7):940-52.

15 El-Khoueiry AB, Sangro B, Yau T, Crocenzi TS, Kudo M, Hsu C, et al. Nivolumab in patients with advanced hepatocellular carcinoma (CheckMate 040): an open-label, non-comparative, phase 1/2 dose escalation and expansion trial. Lancet. 2017 Jun;389(10088):2492-502.

16 Zhu AX, Kang YK, Yen CJ, Finn RS, Galle PR, Llovet JM, et al.; REACH-2 study investigators. Ramucirumab after sorafenib in patients with advanced hepatocellular carcinoma and increased $\alpha$-fetoprotein concentrations (REACH-2): a randomised, double-blind, placebo-controlled, phase 3 trial. Lancet Oncol. 2019 Feb;20(2):28296.

17 Matthieu Komorowski JR. Markov Models and Cost Effectiveness Analysis: Applications in Medical Research.; Secondary Analysis of Electronic Health Records. Cambridge: Springer; 2016. pp. 351-67.

18 Agarwal M, Thareja N, Benjamin M, Akhondi A, Mitchell GD. Tyrosine Kinase Inhibitor-Induced Hypertension. Curr Oncol Rep. 2018 Jun;20(8):65.

19 Tunis SL. A cost-effectiveness analysis to illustrate the impact of cost definitions on results, interpretations and comparability of pharmacoeconomic studies in the US. Pharmacoeconomics. 2009;27(9):735-44.

20 Le QA. Structural Uncertainty of Markov Models for Advanced Breast Cancer: A Simulation Study of Lapatinib. Med Decis Making. 2016 Jul;36(5):629-40.

21 Sarfaty M, Leshno M, Gordon N, Moore A, Neiman V, Rosenbaum E, et al. Cost Effectiveness of Nivolumab in Advanced Renal Cell Carcinoma. Eur Urol. 2018 Apr;73(4):628-34. 
22 Bae YH, Mullins CD. Do value thresholds for oncology drugs differ from nononcology drugs? J Manag Care Spec Pharm. 2014 Nov;20(11):1086-92.

23 Azimi NA, Welch HG. The effectiveness of cost-effectiveness analysis in containing costs. J Gen Intern Med. 1998 Oct;13(10):664-9.

24 Goldstein DA, Chen Q, Ayer T, Howard DH, Lipscomb J, El-Rayes BF, et al. First- and second-line bevacizumab in addition to chemotherapy for metastatic colorectal cancer: a United States-based cost-effectiveness analysis. J Clin Oncol. 2015 Apr;33(10):1112-8.

25 Kobayashi M, Kudo M, Izumi N, Kaneko S, Azuma M, Copher R, et al. Cost-effectiveness analysis of lenvatinib treatment for patients with unresectable hepatocellular carcinoma (uHCC) compared with sorafenib in Japan. J Gastroenterol. 2019 Jun;54(6):558-70.

26 Motevalli MH, Taheri FPS. Cost-effectiveness of sorafenib for hepatocellular carcinoma: a systematic review. Pharm Pharm Res Open Access J. 2019;1:14-21.

27 Shlomai A, Leshno M, Goldstein DA. Regorafenib treatment for patients with hepatocellular carcinoma who progressed on sorafenib-A cost-effectiveness analysis. PLoS One. 2018 Nov;13(11):e0207132.

28 Parikh ND, Singal AG, Hutton DW. Cost effectiveness of regorafenib as second-line therapy for patients with advanced hepatocellular carcinoma. Cancer. 2017 Oct;123(19):3725-31.

29 Soto-Perez-de-Celis E, Aguiar PN, Cordón ML, Chavarri-Guerra Y, Lopes GL. Cost-Effectiveness of Cabozantinib in the Second-Line Treatment of Advanced Hepatocellular Carcinoma. J Natl Compr Canc Netw. 2019 Jun; 17(6):669-75.

30 Goldstein DA, Gordon N, Davidescu M, Leshno M, Steuer CE, Patel N, et al. A Phamacoeconomic Analysis of Personalized Dosing vs Fixed Dosing of Pembrolizumab in Firstline PD-L1-Positive Non-Small Cell Lung Cancer. J Natl Cancer Inst. 2017 Nov;109(11):109.

31 Mukherjee S, Ibrahimi S, Machiorlatti M, Roman D, Saleem R, Hassan A, et al. Personalized Dosing Versus Fixed Dosing of Immune Checkpoint Inhibitors: A Cost Analysis Study. Am J Ther. 2018 Nov/Dec;25(6):e767-8.

32 Levy J, Rosenberg M, Vanness D. A Transparent and Consistent Approach to Assess US Outpatient Drug Costs for Use in Cost-Effectiveness Analyses. Value Health. 2018 Jun;21(6):677-84.

33 Mattingly TJ 2nd, Levy JF, Slejko JF, Onwudiwe NC, Perfetto EM. Estimating Drug Costs: How do Manufacturer Net Prices Compare with Other Common US Price References? Pharmacoeconomics. 2018 Sep;36(9):1093-9.

34 Suh DC, Powers CA, Barone JA, Shin H, Kwon J, Goodin S. Full costs of dispensing and administering fluorouracil chemotherapy for outpatients: A microcosting study. Res Social Adm Pharm. 2010 Sep;6(3):246-56.

35 Finn RS, Ryoo B-Y, Merle P, Kudo M, Bouattour M, et al. Pembrolizumab as Second-Line Therapy in Patients with Advanced Hepatocellular Carcinoma in KEYNOTE-240: A Randomized, Double-Blind, Phase III Trial. J Clin Oncol. 2020 Jan 20;38(3):193-202.

36 Yau T, Park JW, Finn RS, Cheng A, Mathurin P, Edeline J, et al. CheckMate 459: a randomized, multi-center phase III study of nivolumab (NIVO) vs sorafenib (SOR) as first-line (1L) treatment in patients (pts) with advanced hepatocellular carcinoma (aHCC). Ann Oncol. 2019; 30(suppl_5):851-934.

37 Harding JJ, Nandakumar S, Armenia J, Khalil DN, Albano M, Ly M, et al. Prospective Genotyping of Hepatocellular Carcinoma: Clinical Implications of Next-Generation Sequencing for Matching Patients to Targeted and Immune Therapies. Clin Cancer Res. 2019 Apr;25(7):2116-26. 